\title{
Ice on Suspension Bridge Cables
}

\author{
Poul G. Huorth ${ }^{1}$, Viktor Bue Luungdahl ${ }^{2}$, Steffen Madsen $^{2}$, Angelos \\ Ikonomakis ${ }^{1}$, Frederik Listov-Saabye Pedersen ${ }^{1}$, Colin P. Please ${ }^{3}$ \\ and Matthew DaVid Shirley ${ }^{3}$ \\ ${ }^{1}$ Technical University of Denmark, Lyngby, DK \\ ${ }^{2}$ University of Southern Denmark, Odense, DK \\ ${ }^{3}$ University of Oxford, Oxford, UK \\ (Communicated to MIIR on November 10, 2021)
}

Study Group: Falling Ice, ESGI 160, Copenhagen, Denmark, November 10, 2021.

Communicated by: Poul G. Hjorth

Industrial Partner: Light Bureau

Presenter: Thim Nørgaard Andersen

Team Members: T.N. Andersen; P.G. Hjorth; V.B. Ljungdahl; S. Madsen; A. Ikonomakis; F.L.S. Pedersen; C.P. Please; M.D. Shirley

Industrial Sector: Construction; Environment.

Tools: Heat diffusion, Stefan problems, adhesion models

Key Words: Ice shedding, suspension bridges, ice accretion

MSC2020 Codes: 74N25, 74R99, 80A19, 80A22

\section{Summary}

In this report we study mathematical models for predicting when ice may form and fall from vertical steel hangers of suspension bridges down onto the road below. This is an important problem to study, not only because of the economic costs related to closing a bridge due to the risk of falling ice, but also the human cost if the bridge is still open to traffic when ice falls down. In the report we present two main categories of models for predicting falling times: 1) models based on heat transfer from the surrounding air, and 2) models based on heating due to radiation from the sun. A flow chart is furthermore presented together with tables for determining which model to use and quickly estimating time of failure based on a set of simple conditions. 


\section{Introduction}

On suspension bridges in colder climates, such as the Great Belt Bridge in Denmark, ice can form on the vertical hanger cables that hold up the road. This ice has previously been observed to fall from the cable onto the road below. This is an important problem, not only because of the economic cost that closing the bridge when this behaviour is expected incurs, but the human cost if the bridge is still open to traffic when it happens.

In this report we are interested in predicting when ice may form and fall from the vertical steel hangers of a suspension bridge. There are two main types of ice that form on a hanger, called rime and glaze. The main difference is the amount of air within the ice. With rime there is no water layer adjacent to the freezing and hence many air pockets remain in the ice, while glaze has a water film that freezes and hence has few air bubbles. The ice forms either from precipitation (either as freezing rain or as wet snow), or from fog and mist.

There appeared to be two-stages in behaviour of the ice that need considering. Firstly is the creation of the ice layer. Here the composition, shape and size of the ice layer need to be determined. Secondly is the fracturing phase. Here we need to identify the stresses and deformations that will separate chunks of ice from the deposited layer. These two steps occur sequentially and behaviour during the second stage may be closely determine by the first stage.

\section{Literature review}

There is an extensive literature on the first stage of the process where an ice layer is created. For example various researchers in the past have proposed models for how ice is formed around cables [4, 14, 22], or aircraft wings [1, 6]. There are also papers in the literature trying to understand how ice is formed on surfaces in general [20, 21] and consequently, the ice adhesion mechanisms are discussed in $[5,7,15]$. The literature on the second stage is much smaller but includes [13], where L. Kollár and M. Farzaneh model sudden ice shedding from conductor bundles. There is some recent work dealing with how the shapes of ice accretion affect the aerodynamics on the inclined stay cables and straddling hangers of suspension bridges [8, 19] and how ice creates additional friction in surfaces it adheres to $[11,16]$.

In this report, we mainly focus on modeling how ice is shedding from vertical steel hangers of suspension bridges. There has been an initial review in the past by K. Kleissl and C. Georgakakis [12] concerning ice accretion and de-icing systems to prevent big chunks of ice falling on passing automobiles. There are a number of researchers considering how a coating could be created for cables with anti-icing features that would prevent such ice growth. This remains an unsolved manufacturing and engineering problem involving material science as discussed in [17]. But there are solutions where, when ice shedding occurs, the ice is less chunky making the phenomenon less dangerous [17, 18]. 


\section{Possible mechanisms for fracturing stage}

We discussed many different mechanisms that might cause ice to become separated from the hanger and nearby ice. These mechanisms involve both thermal effects and mechanics.

- After the ice is formed there may be changes in heat transfer at the air-ice surface due to surface conditions or wind that cause the ice to melt and lose strength.

- Solar radiation may melt the ice and reduce adhesion to the hanger.

- The hanger may conduct heat from lower parts of the bridge

- The ice may be unable to withstand stresses created due to gravity, vibrations or expansion.

- The ice may begin to melt and this may alter the physical properties of the ice (eg: elastic, yield stress, adhesion etc).

- Changes in temperature may expand of contract the steel hanger and the ice deferentially creating stress.

\section{Estimate of maximum size of ice buildup}

To give a simple estimate of the amount of ice that can form we assume that the only forces acting on the ice are adhesion to the hanger and gravity (hence we are neglecting aspects such as wind stresses). The paper [15] gives experimental measurements of the fracture energy on an ice-steel interface as $1.1 \mathrm{~J} / \mathrm{m}^{2}$, which we will denote by $W_{a}$. They define the work spent moving the ice as

$$
W_{s}=\int_{0}^{\delta x} F(x) \mathrm{d} x
$$

where $F(x)$ is the force applied at position $x$ and $\delta x$ is the amount the ice must be moved to separate. By setting $W_{s}=W_{a}$ we can calculate the maximum sized ice chunk that can be supported.

Assume the ice formation is perfectly circular and centered on the center of the cable. Let $h$ be the height of a cable, $r_{1}$ the radius of the cable, and $r_{2}$ be the radius of the ice buildup from the centre of the cable. The surface area of the ice is given by

$$
\text { Surface area }=2 \pi r_{1} h
$$

and the mass of ice built up is

$$
\text { Mass ice }=\rho \pi h\left(r_{2}^{2}-r_{1}^{2}\right)
$$

where $\rho$ is density of ice. Hence we get

$$
2 \pi r_{1} h W_{a}=\rho \pi h\left(r_{2}^{2}-r_{1}^{2}\right) g \delta x
$$

Simplifying

$$
r_{2}^{2}=\frac{2 W_{a} r_{1}}{\rho g \delta x}+r_{1}^{2}
$$

We use $W_{a}=1.1 \mathrm{~J} / \mathrm{m}^{2}, \rho=900 \mathrm{~kg} / \mathrm{m}^{3}, g=10 \mathrm{~m} / \mathrm{sec}^{2}$ and $r_{1}=5 \times 10^{-2} \mathrm{~m}, r_{1}=5 \mathrm{~cm}$. The value of $\delta x$ is hard to characterise, but $\delta x=1 \mathrm{~mm}$ gives an ice width of $7 \mathrm{~cm}$. Note that whatever shape the ice forms, the maximum mass of ice that can be supported will 


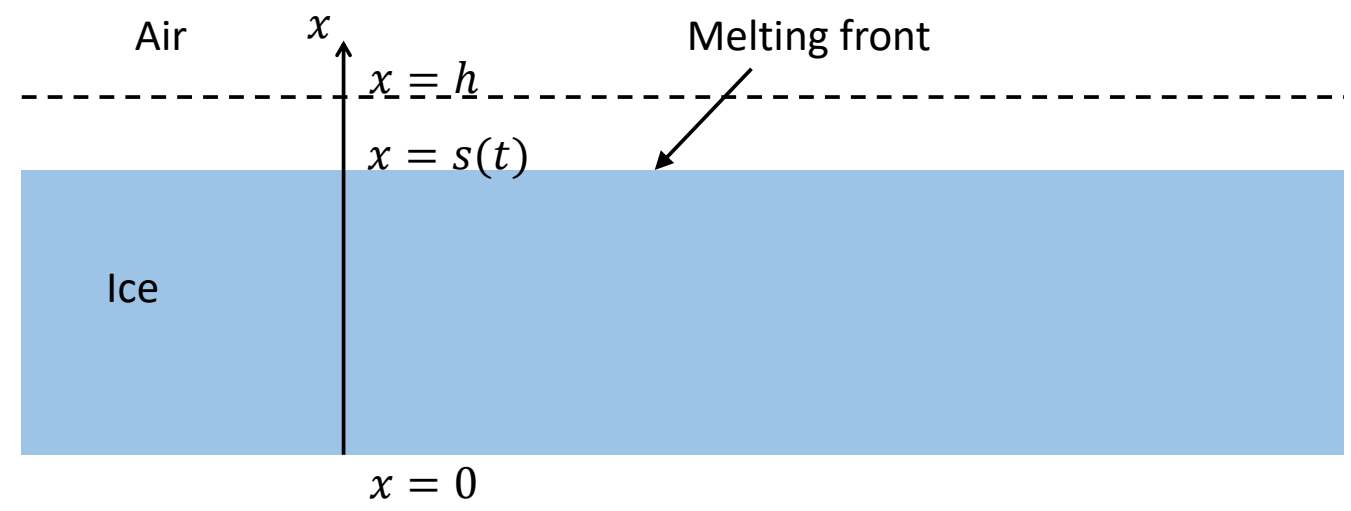

Figure 1. The domain considered for the one-dimensional melting due to air temperature problem with center-line at $x=0$ and ice-air interface at $x=s(t)$.

remain unchanged, provided the whole surface of the cable is coated in ice. For the values above this gives that the cable can support at most $33 \mathrm{~kg} / \mathrm{m}$ of ice.

\section{Melting due to air temperature and surface absorption of radiation in one-dimension}

We now consider a simple model for the melting of the ice. Our initial approach is to take a simple 1-D geometry and determine how long it takes to completely melt some ice if heat transfer from the air is the only mechanism. We shall treat the ice as a homogeneous solid material, neglecting the effect of any voids or impurities. Such a model is likely to be appropriate for glazed ice, but not for rime ice which has many air pockets. In the subsequent section we shall generalise the model to an arbitrarily shaped two-dimensional deposit of ice on a cable and include the effect of surface absorption of solar radiation, i.e. heating from the sun.

\subsection{Model}

We shall consider an infinite slab of ice with finite thickness $s(t)$ at time $t$. Assuming diffusion is the only heat transfer mechanism within the ice, we can model the temperature $T$ using the heat equation

$$
\rho c_{p} \frac{\partial T}{\partial t}=\frac{\partial}{\partial x}\left(k \frac{\partial T}{\partial x}\right), \quad \text { in } 0<x<s(t)
$$

where $\rho, c_{p}$, and $k$ are the density, specific heat capacity (at constant pressure), and thermal conductivity of the ice respectively. The boundary condition at the interface between the air and ice, $x=s(t)$ has two different behaviours: whilst the temperature of the interface is below the melting point the appropriate boundary condition is continuity of heat flux, namely, if $T<T_{\text {melt }}$ then at $x=h$

$$
H\left(T-T_{a}\right)=k \frac{\partial T}{\partial x}
$$


where $T_{\text {melt }}$ and $T_{a}$ are the melting temperature and temperature of the air respectively, $H$ is the heat transfer coefficient between the ice and the air. When the surface reaches the melting temperature we have to change the boundary condition to account for melting. Assuming any water that forms from melting either falls or is advected away by the air, we can assume that the ice-air interface $x=s(t)$, simply moves to account for the loss of material from the ice. The appropriate boundary condition now comes from assuming a jump in the heat flux across the boundary equal to the amount of energy required to melt the material on the boundary,

$$
T=T_{\text {melt }}, \quad \text { and } \quad \rho L \frac{\mathrm{d} s}{\mathrm{~d} t}=H\left(T_{\text {melt }}-T_{a}\right)-k \frac{\partial T}{\partial x}
$$

where $L$ is the specific latent heat of ice melting. Note that we require $T_{a}>T_{m e l t}$, so that melting occurs. This is a common type of boundary condition for free boundary problems, see [3] for more details of their derivation.

For simplicity, we shall ignore any effects due to the cable, and assume the behaviour of the ice to be symmetric about $x=0$, meaning

$$
\frac{\partial T}{\partial x}=0 \quad \text { at } x=0
$$

The initial conditions are

$$
s(t)=h, \quad T(x)=T_{\text {init }}(x) \quad \text { at } t=0,
$$

so that $h$ is the initial height of the ice.

\subsection{Nondimensionalisation}

We now nondimensionalise the problem in order to systematically reduce the number of parameters in the problem and identify the most important physical processes. We start by scaling the variables as follows

$$
\begin{array}{r}
x=h \hat{x}, \quad s=h \hat{s}, \\
T=T_{\text {melt }}+\Delta T \hat{T}, \quad t=t_{\text {ref }} \hat{t}
\end{array}
$$

Here, we have left the timescale, $t_{r e f}$, undefined and will subsequently choose a natural timescale in the problem related to the heat flux and the latent heat (other timescale, such as the diffusion time across the ice are also possible choices and would not change the analysis).

The governing equation (5.1) becomes

$$
\frac{\partial \hat{T}}{\partial \hat{t}}=\frac{k t_{r e f}}{\rho h^{2} c_{p}} \frac{\partial^{2} \hat{T}}{\partial x^{2}}
$$

with boundary conditions,

$$
\frac{\partial T}{\partial x}=0 \quad \text { at } \hat{x}=0
$$

and, if $\hat{T}<\hat{T}_{\text {melt }}$ at $\hat{x}=1$

$$
\frac{H \Delta T t_{r e f}}{\rho L h}\left(\hat{T}-\hat{T}_{a}\right)=\frac{k t_{r e f}}{\rho h^{2} c_{p}} \frac{c_{p} \Delta T}{L} \frac{\partial \hat{T}}{\partial \hat{x}} \quad \text { at } \hat{x}=\hat{s}(\hat{t}),
$$




\begin{tabular}{c|c} 
Parameter & Value \\
$\Delta T$ & $5 \mathrm{~K}$ \\
$k$ & $2.22 \mathrm{~W} / \mathrm{mK}$ \\
$L$ & $334 \times 10^{3} \mathrm{~J} / \mathrm{kg}$ \\
$c_{p}$ & $2.0 \times 10^{3} \mathrm{~J} / \mathrm{kgK}$ \\
$h$ & $5 \times 10^{-2} \mathrm{~m}$ \\
$H$ & $30 \mathrm{~W} / \mathrm{m}^{2} \mathrm{~K}$ \\
$\rho$ & $900 \mathrm{~kg} / \mathrm{m}^{3}$
\end{tabular}

Table 1. Typical parameter values used for melting due to air heating problem.

otherwise

$$
\begin{aligned}
\hat{T}=\hat{T}_{\text {melt }} & \text { at } \hat{x}=\hat{s}(\hat{t}), \\
\frac{\mathrm{d} \hat{s}}{\mathrm{~d} \hat{t}}=\frac{H \Delta T t_{r e f}}{\rho L h}\left(\hat{T}-\hat{T}_{a}\right)-\frac{k t_{r e f}}{\rho h^{2} c_{p}} \frac{c_{p} \Delta T}{L} \frac{\partial \hat{T}}{\partial \hat{x}} & \text { at } \hat{x}=\hat{s}(\hat{t}),
\end{aligned}
$$

where $\hat{T}_{\text {melt }}=0$, and $\hat{T}_{a}=\left(T_{a}-T_{\text {init }}\right) / \Delta T$ are the nondimensional melting and air temperatures.

Based on this nondimensionalisation there are two timescales in the problem

$$
\frac{\rho h^{2} c_{p}}{k}=2027 \mathrm{sec} \approx 30 \mathrm{~min} \quad \text { and } \quad \frac{\rho L h}{H \Delta T}=100200 \mathrm{sec} \approx 28 \text { hours. }
$$

associated with diffusion within the ice and the speed at which the interface changes, with their values calculated from table 1 . As the time-scale associated with the movement of the interface is longest we choose it as our temporal scaling, setting

$$
t_{\text {ref }}=\frac{\rho L h}{H \Delta T} \text {. }
$$

The problem therefore becomes

$$
\frac{\partial \hat{T}}{\partial \hat{t}}=\frac{1}{\mathrm{NuSt}} \frac{\partial^{2} \hat{T}}{\partial \hat{x}^{2}} \quad \text { in } 0<\hat{x}<\hat{s}(\hat{t})
$$

with boundary conditions

$$
\frac{\partial T}{\partial \hat{x}}=0 \quad \text { at } \hat{x}=0
$$

and, for $T<T_{\text {melt }}$

$$
\hat{T}-\hat{T}_{a}=\frac{1}{\mathrm{Nu}} \frac{\partial \hat{T}}{\partial \hat{x}} \quad \text { at } \hat{x}=\hat{s}(\hat{t})
$$

otherwise

$$
\begin{aligned}
\hat{T} & =\hat{T}_{\text {melt }} & \text { at } \hat{x} & =\hat{s}(\hat{t}), \\
\frac{\mathrm{d} \hat{s}}{\mathrm{~d} \hat{t}} & =\hat{T}-\hat{T}_{a}-\frac{1}{\mathrm{Nu}} \frac{\partial \hat{T}}{\partial \hat{x}} & \text { at } \hat{x} & =\hat{s}(\hat{t}),
\end{aligned}
$$

where

$$
\mathrm{St}=\frac{c_{p} \Delta T}{L}, \quad \mathrm{Nu}=\frac{H h}{k} .
$$

where St is called the Stefan number, (in some texts the Stefan number defined as a reciprocal) and $\mathrm{Nu}$ is the Nusselt number. The Stefan number is the ratio of sensible to 
latent heat, and the Nusselt number the ratio of of convective to conductive heat transfer at the boundary. Based on the parameter values in table 1, typical values would be

$$
\mathrm{St}=0.06, \quad \mathrm{Nu}=0.75
$$

It would therefore seem appropriate to examine the solution in the limit $\mathrm{St} \rightarrow 0$.

\subsection{The Small Stefan number limit}

When we consider the limit where the Stefan number is small (ie. when the latent heat dominates over the sensible heat) we find the problem has two regimes. First there is a rapid transient where the temperature in the ice first reaches the melting point of the ice. There is then a long regime where the temperature in the ice remains near the melting point everywhere and the surface moves primarily due to a balance between the heat flux from the air and the latent heat.

\subsubsection{Short time behaviour}

There is a transient, on a timescale where $\hat{t}=O(1 / \mathrm{St})$, where the temperature adjust from its initial condition to a long time behaviour. In the first part of this transient the temperature is above melting everywhere and so

$$
\frac{\partial \hat{T}}{\partial \hat{t}}=\frac{1}{\mathrm{NuSt}} \frac{\partial^{2} \hat{T}}{\partial \hat{x}^{2}} \quad \text { in } 0<\hat{x}<\hat{s}(\hat{t})
$$

with boundary conditions

and

$$
\frac{\partial T}{\partial \hat{x}}=0 \quad \text { at } \hat{x}=0
$$

$$
\hat{T}-\hat{T}_{a}=\frac{1}{\mathrm{Nu}} \frac{\partial \hat{T}}{\partial \hat{x}} \quad \text { at } \hat{x}=\hat{s}(\hat{t}), \quad \text { at } \hat{x}=1,
$$

This is a classical heat diffusion problem. The solution can solved analytically. This early part of the behaviour can be extended to more complex geometries. for example circular regions would be appropriate for representing thin ice on a cable and also include solar heating that depends on position around the cable. Analytical solutions to such cases can be found in the extensive examples in [2].

Because this is a rapid transient $\hat{s}(t)$ remains very close to its original position $x=$ 1. Hence, once the melting temperature is reached on the surface, the boundary will change to $\hat{T}=T_{\text {melt }}$ and the solution to the diffusion equation can then be put into the heat conduction condition to determine the small distance $s(t)$ starts to move and the temperature everywhere tends to the melting temperature.

\subsubsection{Long time behaviour}

For the longer times the problem is a classical Stefan problem. Such problems are discussed in detail in [3]. Such problems are best solved using numerical the "Enthalpy method" but here we present an approximate analytical solution that exploits the small Stefan number. 
We consider the behaviour for $\hat{t}=O(1)$ and $\mathrm{St} \rightarrow 0$. Then, assuming that both $\hat{T}$ and $\hat{s}$ can be expanded in the form

$$
\hat{T}(\hat{x}, \hat{t}, \mathrm{St}) \sim \hat{T}_{0}(\hat{x}, \hat{t})+\mathrm{St} \hat{T}_{1}(\hat{x}, \hat{t})+\mathrm{St}^{2} \ldots,
$$

at lowest order, the problem is

$$
0=\frac{\partial^{2} \hat{T}_{0}}{\partial \hat{x}^{2}} \quad \text { in } 0<\hat{x}<\hat{s}(\hat{t})
$$

with boundary conditions

$$
\begin{aligned}
\frac{\partial \hat{T}_{0}}{\partial \hat{x}} & =0 & & \text { at } \hat{x}=0 \\
\hat{T}_{0} & =\hat{T}_{\text {melt }} & & \text { at } \hat{x}=\hat{s}(\hat{t}), \\
\frac{\mathrm{d} \hat{s}}{\mathrm{~d} \hat{t}} & =\hat{T}_{0}-\hat{T}_{a}-\frac{1}{\mathrm{Nu}} \frac{\partial \hat{T}_{0}}{\partial \hat{x}} & & \text { at } \hat{x}=\hat{s}(\hat{t}),
\end{aligned}
$$

and initial condition

$$
\hat{s}(0)=1 \text {. }
$$

Solving the temperature equation and using the first two boundary conditions we see that $\hat{T}_{0}=\hat{T}_{\text {melt }}$ for all $x$ and $t$. Putting this solution into the third boundary condition, the Stefan condition, this becomes an ODE for $s$ given by

$$
\frac{\mathrm{d} \hat{s}}{\mathrm{~d} \hat{t}}=\hat{T}_{m e l t}-\hat{T}_{a}
$$

Integrating, and using initial condition for $\hat{s}$,

$$
\hat{s}(\hat{t})=\left(\hat{T}_{\text {melt }}-\hat{T}_{a}\right) \hat{t}+1
$$

As $\hat{T}_{\text {melt }}-\hat{T}_{a}<0$, this means ice region shrinking. Letting $\hat{t}^{*}$ be the time such that $\hat{s}\left(\hat{t}^{*}\right)=0$, we see

$$
\hat{t}^{*}=\frac{1}{\hat{T}_{a}-\hat{T}_{\text {melt }}} .
$$

In dimensional time we find that the time until the block of ice is completely melted is given by

$$
t^{*}=t_{\text {ref }} \hat{t}^{*}=\frac{\rho L h}{H \Delta T} \frac{1}{\hat{T}_{a}-\hat{T}_{\text {melt }}}=\frac{\rho L h}{H\left(T_{a}-T_{\text {melt }}\right)}=\approx 27 \text { hours }
$$

and the numerical value here is derived using the parameter values we have given earlier. This gives a simple expression, based on easily measured physical quantities, for the melting time of a block of ice in the physically relevant situation where the Stefan number is small.

\subsubsection{Extension to variable ambient temperature}

This analysis trivially extends to an ambient temperature that varies with time $T_{a}(t)$. Then, in the small Stefan number limit,

$$
\frac{\mathrm{d} \hat{s}}{\mathrm{~d} \hat{t}}=\hat{T}_{m e l t}-\hat{T}_{a}(\hat{t}),
$$




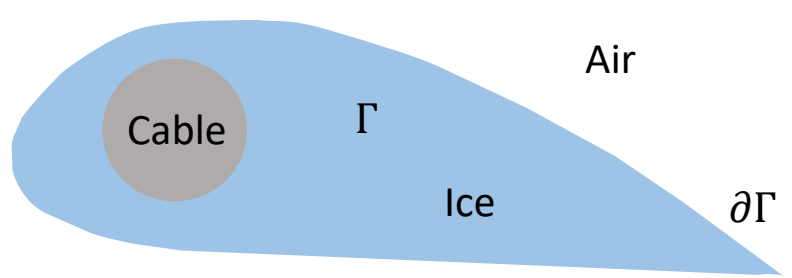

Figure 2. The domain $\Gamma$ considered for the arbitrary two-dimensional problem, including the cable and ice-air interface $\partial \Gamma$.

which (using the initial condition for $\hat{s}$ ) integrates to

$$
\hat{s}(\hat{t})=\hat{T}_{\text {melt }} \hat{t}-\int_{0}^{\hat{t}} \hat{T}_{a}(\tilde{t}) \mathrm{d} \tilde{t}+1
$$

We cannot explicitly solve for the melting time $\hat{t}^{*}$ as in (5.34) but we can find it using an iterative algorithm based on a simple numerical integration method.

Let $\hat{T}_{a_{1}}, \hat{T}_{a_{2}}, \hat{T}_{a_{3}}, \ldots$ be a sequence of temperatures observed at times $t_{1}, t_{2}, t_{3}, \ldots$ We then construct the sequence $s_{1}, s_{2}, s_{3}, \ldots$ which estimates the thickness of the ice at the corresponding times $t_{1}, t_{2}, t_{3}, \ldots$ as follows:

$$
s_{1}=1 \quad s_{n+1}=s_{n}+\left(t_{n+1}-t_{n}\right)\left(\hat{T}_{m e l t}-\hat{T}_{a_{n}}\right)
$$

Note that if the temperature is constant $\left(\hat{T}_{a_{1}}=\hat{T}_{a_{2}}=\cdots=\hat{T}_{a}\right)$ then we get

$$
s_{n}=\left(\hat{T}_{m e l t}-\hat{T}_{a}\right)\left(t_{n}-t_{1}\right)+1
$$

which is the discrete version of (5.32). Whenever some $s_{n} \leq 0$ is encountered, we expect that $t_{n-1}<\hat{t}^{*} \leq t_{n}$, so we set

$$
\hat{t}^{*}=\frac{s_{n} t_{n-1}-s_{n-1} t_{n}}{s_{n}-s_{n-1}}
$$

This corresponds to finding the root of the straight line drawn through the two points $\left(t_{n-1}, s_{n-1}\right)$ and $\left(t_{n}, s_{n}\right)$.

\section{Melting of an arbitrarily shaped ice block due to air temperature and surface absorption of radiation}

We now extend the analysis of the previous section to the application relevant crosssection of ice deposited on a cable. We also outline the extension to surface absorption of solar radiation.

\subsection{Model}

The ideas of the previous model can be extended to consider a cross-sectional region of ice and steel cable and how it melts due to air flow. We can write the more general 
diffusion equation in two dimensions with a moving surface.

$$
\rho c_{p} \frac{\partial T}{\partial t}=\nabla(k \nabla T), \quad \text { in } \Gamma
$$

where $\rho, c_{p}$, and $k$ may depend spatially depending on the position of the ice and the steel cable and $\Gamma$ is the region of space containing both the cable and the ice. Denoting the ice-air boundary by $\partial \Gamma$, boundary conditions for the problem are at points on the boundary where $T<T_{\text {melt }}$ the boundary remains fixed in space and

$$
H\left(T-T_{a}\right)=k \nabla T \cdot \mathbf{n}+B q_{s o l}
$$

otherwise, on the moving boundary

$$
T=T_{\text {melt }}, \quad \rho L \mathbf{v} \cdot \mathbf{n}=H\left(T-T_{a}\right)-k \nabla T \cdot \mathbf{n}+B q_{\text {sol }},
$$

where $\mathbf{v}$ is the velocity of the outer surface $\partial \Gamma, \mathbf{n}$ is the outward facing normal to $\partial \Gamma$ and $B$ is the proportion of solar radiation $q_{s o l}$ absorbed by the surface. We assume the remainder of the radiation is reflected and no internal heating occurs.

For some results of how to treat evolution of a moving surface in more than onedimension we follow [10] page 14. First we introduce the function $f(\mathbf{x}, t)$ which implicitly defines the location of $\partial \Gamma$ at time $t$ via $f(\mathbf{x}, t)=0$. Then the outward-pointing unit normal $\mathbf{n}=\nabla f /|\nabla f|$. Now any point $\mathbf{x}_{B}(t)$ in $\partial \Gamma$ at time $t$ must satisfy

$$
\frac{\mathrm{d} \mathbf{x}_{B}}{\mathbf{d} t}=v, \quad \text { and } \quad f\left(\mathbf{x}_{B}(t), t\right)=0,
$$

hence, by differentiating the second equation and substituting in the first we obtain

$$
\frac{\partial f}{\partial t}+\mathbf{v} \cdot \nabla f=0, \quad \Leftrightarrow \frac{\partial f}{\partial t}+\mathbf{v} \cdot \mathbf{n}=0,
$$

which gives the relationship between the position of the boundary $f$ and its velocity and every point along the boundary.

Finally, for initial conditions of the problem we take

$$
T=T_{\text {init }}(x), \quad \text { and } f(\mathbf{x}, t)=f_{0}(\mathbf{x}) .
$$

\subsection{Nondimensionalisation}

We nondimensionalise using the same scalings as before,

$$
\begin{array}{r}
\mathbf{x}=h \hat{\mathbf{x}}, \quad \mathbf{v}=\frac{h}{t_{r e f}}, \hat{\mathbf{v}}, \quad T=T_{\text {melt }}+\Delta T \hat{T}, \\
t=\frac{\rho_{r e f} L h}{H \Delta T} \hat{t}, \quad \rho=\rho_{r e f} \hat{\rho}, \quad c_{p}=c_{p, r e f} \hat{c}_{p}, \quad k=k_{r e f} \hat{k},
\end{array}
$$

Note here we are now allowing the thermodynamic parameters, of density, specific heat and conductivity to vary spatially, to account for regions of ice and steel and have introduced $\hat{\rho}, \hat{c}_{p}$, and $\hat{k}_{r e f}$ as dimensionless functions expressing this dependence. As in the one-dimensional analysis we have chosen the timescale of heat transfer from air to ice as our dominant timescale. The inclusion of solar radiation technically introduces an additional timescale into the problem, but we shall assume it is always on the same time scale of heat transfer from the air. 
This then gives us the dimensionless problem (we drop the hat notation for convenience)

with boundary conditions

$$
\operatorname{NuSt} \rho c_{p} \frac{\partial T}{\partial t}=\nabla(k \nabla T), \quad \text { in } \Gamma
$$

$$
\begin{aligned}
& 0=T-T_{a}-\frac{1}{\mathrm{Nu}} k \nabla T \cdot \mathbf{n}+\beta q_{\text {sol }} \quad \text { on } \partial \Gamma, \quad \text { where } T<T_{\text {melt }}, \\
& \rho \mathbf{v} \cdot \mathbf{n}=T-T_{a}-\frac{1}{\mathrm{Nu}} k \nabla T \cdot \mathbf{n}+\beta q_{\text {sol }} \quad \text { on } \partial \Gamma, \quad \text { where } T=T_{\text {melt }} .
\end{aligned}
$$

where,

$$
\mathrm{St}=\frac{c_{p, r e f} \Delta T}{L}, \quad \mathrm{Nu}=\frac{H h}{k_{r e f}}, \quad \beta=\frac{B q_{\text {sol }, r e f}}{H \Delta T}
$$

where as before St and $\mathrm{Nu}$ are the Stefan and Nusselt numbers, and $\beta$ is the nondimensional ratio of solar heating to convective heating from the air.

\subsection{Small Stefan number}

As before we will consider the St $\rightarrow 0$ limit. We note there is a rapid transient, but focus our analysis on the $t=O(1)$ region. To lowest order we get the equation

$$
0=\nabla(k \nabla T), \quad \text { in } \Gamma
$$

and if we impose the first boundary condition on the surface we find that

$$
T \equiv T_{\text {melt }}
$$

Taking this solution and putting it into the second boundary condition, (6.11), to find

$$
-\rho \frac{\partial f}{\partial t}=\rho \mathbf{v} \cdot \mathbf{n}=T_{m e l t}-T_{a}+\beta q_{\text {sol }}
$$

The condition (6.15) simply states that provided $T_{a}$ and $q_{\text {sol }}$ are fixed in value, the boundary melts at a uniform rate in a direction normal to the surface. For any given initial shape of ice we can readily deduce when the outer boundary first contacts the steel hanger within the ice and this might be a simplest failure criteria for the ice.

\subsection{Bulk heat from the sun: Mushy region model}

Radiation from the sun can be adsorbed as heat either at the interfaces (we have previously looked a the heat adsorbed at the ice/air interface, but it can also occur at the ice/hanger interface) however, there can also be heat absorbed through the depth of the ice. These ideas have been considered elsewhere in the context of heating in glaciers such as [9]. We present some initial ideas related to this but we have not developed the ideas very far. The model is similar to before, but rather than modelling the ice-air interface as sharp, we shall instead assume there is an intermediate "mushy" region as discussed in [3].

We now let $\Gamma_{1}$ represent the region where the ice is solid. The boundary between the solid ice and the mushy region $\partial \Gamma_{1}$ is implicitly specified by $f_{1}(\mathbf{x}, t)=0$. 
The mushy region is $\Gamma_{2}$ and it has an interior boundary $\partial \Gamma_{1}$ with the solid region, and a mush-air boundary $\partial \Gamma_{2}$ implicitly defined by $f_{2}(\mathbf{x}, t)=0$. We therefore have two free boundaries in the model.

In the mush region, we expect the temperature to equal the melting temperature everywhere $T=T_{m e l t}$. Therefore the energy equation describes how heat is supplied to melt the solid. Therefore, letting $\theta$ be the volume fraction of ice, the governing equations are

$$
\begin{array}{rr}
\rho c_{p} \frac{\partial T}{\partial t}=\nabla(k \nabla T)+Q & \text { in } \Gamma_{1}, \\
\rho L \frac{\partial \theta}{\partial t}=Q & \text { in } \Gamma_{2} .
\end{array}
$$

The boundary conditions are more complicated. We require continuity in $\theta$ and $T$ across the ice-mush boundary, meaning

$$
\theta=1, \quad \text { and } T=T_{\text {melt }} \quad \text { on } \partial \Gamma_{1}
$$

while $\theta$ need not equal zero at $\partial \Gamma_{2}$. This possible discontinuity is the same as the sharp interface model. Across the ice-mush interface we must have continuous temperature and $\theta$ and conservation of energy, meaning

$$
\begin{aligned}
T & =T_{\text {melt }}, \\
{[\theta]_{-}^{+} } & =0, \\
{\left[\rho L \theta \mathbf{v}_{1} \cdot \mathbf{n}_{1}+k \nabla T \cdot \mathbf{n}_{1}\right]_{-}^{+} } & =0, \quad \text { on } \partial \Gamma_{2}
\end{aligned}
$$

where the temperature gradient in the mush region, in the final condition, region will be zero.

\section{Heating of a the cable by the sun}

In this section it is estimated how long time it will take for the cable that the ice is stuck on, to exceed a temperature of $0^{\circ} \mathrm{C}$, due to the cable being heated by the sun. Here only the cable is considered and the heating of the ice itself from solar radiation is not considered. Such a model is appropriate for a clear ice, with a small absorption coefficient. The heating of the cable is based on the energy balance between the sun and the cable. It is assumed that when the cable exceeds $0^{\circ} \mathrm{C}$ the ice in contact with the cable will start to melt and lose its adhesion with the cable, thereby causing it to fall. Assuming that the wire is a steel cylinder of length 1 meter the heat capacity of the cable is calculated as follows

$$
C_{c}=\pi r^{2} \rho_{c} c_{p, c}
$$

where $\rho_{c}$ is the density of steel, $c_{p, c}$ is the specific heat capacity of steel, and $C_{c}$ is the heat capacity of a 1 meter long steel cylinder with a radius $r$. We denote the power from the sun $Q_{\text {solar }}$ and the power absorbed by the wire $q_{\text {solar }}$ as

$$
q_{\text {solar }}=Q_{\text {solar }}(1-a)(1-b) \sin (\theta)
$$

where $a$ is the albedo parameter related to reflected energy at the ice surface, $b$ is the fraction of energy absorbed by the ice layer and $\theta$ describes the angel of incidence. We 
denote the target temperature as $T_{c}=0$ and the initial temperature as $T_{i, c}$. The energy balance between the cable and the sun's heating can then be expressed.

$$
\left(T_{i, c}-T_{c}\right) C_{c}=A q_{\text {solar }}
$$

where $A$ is the area of the cable. Since $q_{\text {solar }}$ is the energy per unit time $q_{\text {solar }}=\frac{E_{\text {solar }}}{\Delta t}$, where $E_{\text {solar }}$ is the energy delivered and $\Delta t$ is the amount of time for the cable to reach the required temperature, using this with equation (7.3) it can be rewritten to obtain $\Delta t$.

$$
\Delta t=\frac{\left(T_{i, c}-T_{c}\right) C_{c}}{E_{\text {solar }} A}
$$

Through this the time required to obtain high enough temperatures to melt the ice can be estimated as a function of different parameters.

\section{Applying the Models}

This section presents a method for quickly and intuitively applying the presented models for predicting when ice may fall from a vertical steel hangers of a suspension bridge. The method contains two steps: 1) Look up which of the presented models to apply using a flow chart (Section 8.1), and 2) based on the model, look up related times in a table containing precomputed values (Section 8.2) .

\subsection{Classification system}

In this section we present a flow chart for identifying which of the presented models to apply in a give situation. The flow chart seen in Figure 3, uses four categories and their condition for determining the recommended actions to take e.g. which model to use. These categories are in a hierarchical order: 1) air temperature, 2) day time (sun is up vs. sun is down), 3) elongated shape (yes or no). In the chart we present five groups of possible actions:

- Look up time related to the Stefan model (see Equation 5.34). Potential danger of falling ice.

- Look up time related to the radiation model (see Equation 7.4). Potential danger of falling ice.

- Not critical - keep monitoring the ice, no clear threat of falling ice detected.

- Not included in any of the presented models - the model does not cover this group, this should be covered in future work. Potential danger of falling ice.

- Look up time related to both the Stefan model (see Equation 5.34) and the radiation model (see Equation 7.4), and use the lowest time as a reference. Potential danger of falling ice.

The model will serve as a guide for which actions to take when ice has been detected, and can support the already used experience based method. 


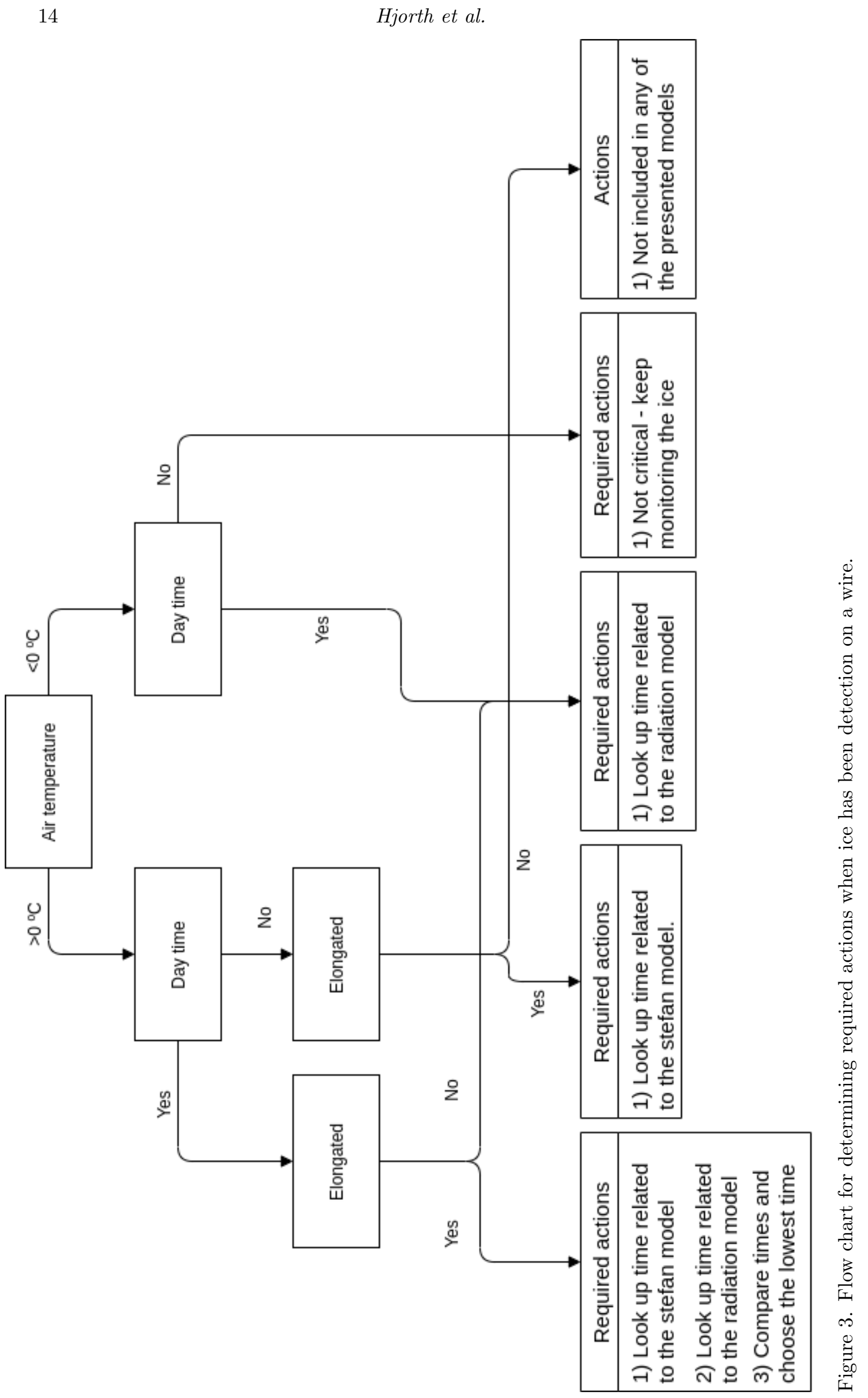




\subsection{Time Tables}

Once the flow chart has been followed and the appropriate model has been identified, the estimated time remaining until the ice falls can be calculated by either i) using the appropriate formula or ii) using a quick estimate of the time from Table 5 or Table 6. The estimates in Table 5 are calculated through equations (5.14) and (5.34) while the iterative algorithm in equation (5.37) is used to estimate the air temperature for a dynamic air temperature profile. However, equation (5.34) can be utilized with a constant air temperature if a dynamic profile is not at hand. To estimate melting of the ice due to heat transfer from the air a sample of air temperatures from a winter month, from a Design Reference Year(DRY) has been used. The data contains the air temperature in Denmark for one year, with hourly time steps. The DRY and a small sample of the data can be seen in Figure 4. The sample seen here is used for estimating the air temperatures $0^{\circ} \mathrm{C} \leq T_{a}<5^{\circ} \mathrm{C}$ while for temperatures $5^{\circ} \mathrm{C} \leq T_{a}<10^{\circ} \mathrm{C}$ the same data has been offset by $+5^{\circ} \mathrm{C}$. The air speed is considered in the calculations through its influence on the
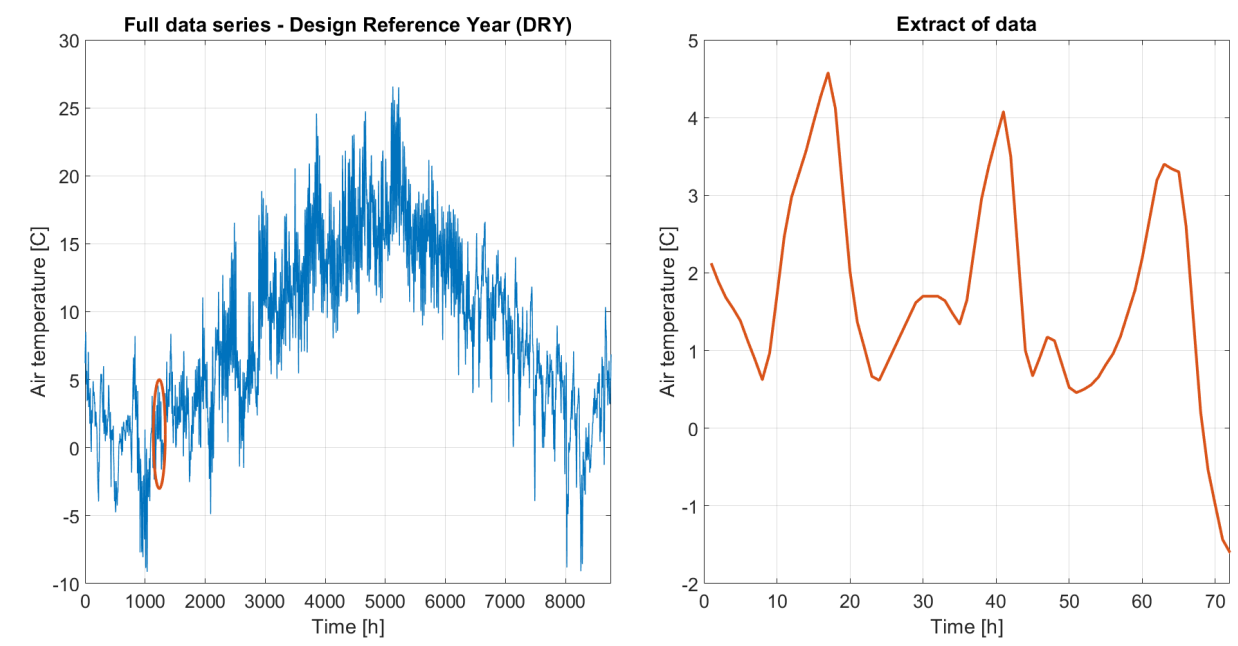

Figure 4. Left: The DRY air temperature profile for the entire year. (red circle = extract) Right: Extract of three days used for simulation of a dynamic air temperature profile.

convective heat transfer coefficient. Here it is assumed that the ice takes the shape of a cylinder and the corresponding correlations are utilized

$$
\begin{gathered}
r=r_{c}+h \\
D=\frac{1}{2} r \\
R e=\frac{\rho_{a} v_{a} D}{\mu_{a}} \\
N u=0.027 P r_{a} R e^{0.805} \\
H=\frac{N u k_{a}}{D}
\end{gathered}
$$




\begin{tabular}{c|c|c|c|c} 
Thermal conductivity & Density & Dynamic viscosity & Specific heat & Prandtl \\
\hline $24.35 \times 10^{-3}\left[\mathrm{~W} / \mathrm{m} \times{ }^{\circ} \mathrm{K}\right]$ & $1.276\left[\mathrm{~kg} / \mathrm{m}^{3}\right]$ & $17.22 \times 10^{-} 6\left[(\mathrm{~N} \times \mathrm{s}) / \mathrm{m}^{2}\right]$ & $1.006\left[\mathrm{~J} / \mathrm{kg} \times{ }^{\circ} \mathrm{K}\right]$ & $0.711[-]$ \\
\hline
\end{tabular}

Table 2. Thermal and fluid properties of air used for estimation of convective heat transfer coefficient.

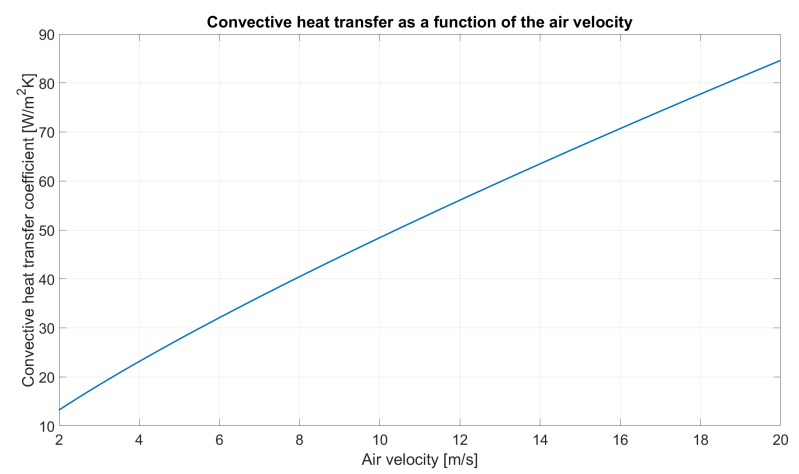

Figure 5. Convective heat transfer coefficient for air velocities between $2 \mathrm{~m} / \mathrm{s}$ and 20 $\mathrm{m} / \mathrm{s}$.

where $r$ is the total radius of the cable including the ice, $r_{c}$ is the radius of the cable, $\mathrm{h}$ is the thickness of the ice, $D$ is the characteristic length, $R e$ is the Reynolds number, $\rho_{a}$ is the density of the air, $v_{a}$ is the air velocity, $\mu_{a}$ is the dynamic viscosity of air, $\operatorname{Pr}_{a}$ is the Prandtl number of air, $H$ is the convective heat transfer coefficient at the air-ice interface, $N u$ is the Nusselt number and $k_{a}$ is the thermal conductivity of the air. Here the density, dynamic viscosity, conductivity and Prandtl number are assumed constant and the utilized properties can be found in Table 2. These properties are temperature dependent to some degree but within the relative small temperature interval that is considered $\left(+-5^{\circ} \mathrm{C}\right)$, this likely represents a very small inaccuracy in the estimation. How the convective heat transfer coefficient varies with air velocity can be seen in Figure 5, showing the importance of this parameter in the melting of the ice.

For Table 6 equations (7.1)-(7.4) are utilized. In Figure 6 how the time required to heat the cable up to $0^{\circ} \mathrm{C}$ as a function of different parameters in the estimate can be seen. The standard values used for this estimation and the values that are varied can be seen in Table 3 and Table 4, respectively.

However, the requirements needed to utilize the tables are of course less restrictive. For Table 6 only the approximate time of day and the approximate cable temperature is needed. If measurement of the cable temperature is not available the night time temperature can be used as an estimate.

Table 5 requires knowledge about the ambient air temperature during when the cable is expected to melt, the approximate wind speed and thickness of the ice at its thinnest point. In the case where one or more of the parameters are not known to the required accuracy, the shortest time period of several table entries are advised to be utilized. 

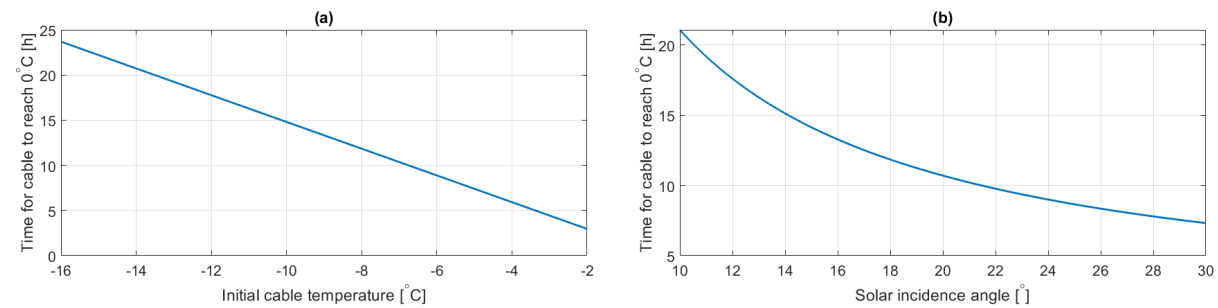

(c)
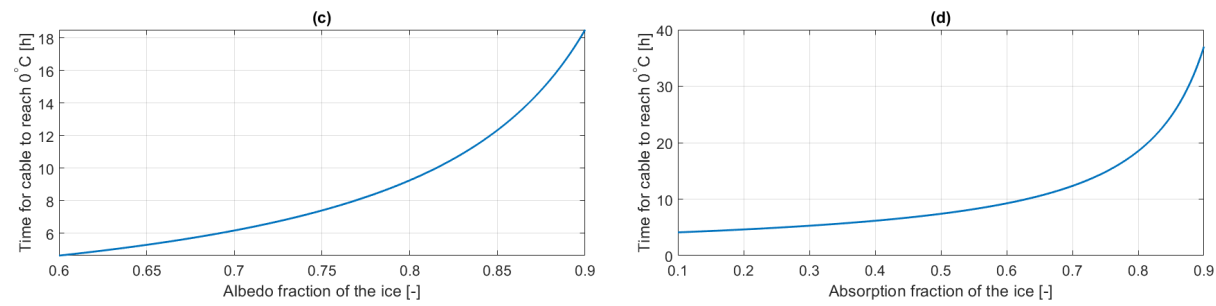

Figure 6. Time to heat up the the hanging cable to $0^{\circ} \mathrm{C}$, as a function of (a) The initial cable temperature, (b) Angle of incidence of the sun, (c) the albedo of the ice and (d) the absorption fraction of the ice.

\begin{tabular}{c|c}
\hline$\rho$ & $8000\left[\mathrm{~kg} / \mathrm{m}^{3}\right]$ \\
$c_{p}$ & $420\left[\mathrm{~J} /\left(\mathrm{kg}{ }^{\circ} K\right)\right]$ \\
$a$ & $0.75[-]$ \\
$b$ & $0.5[-]$ \\
$\theta$ & $20\left[^{\circ}\right]$ \\
$T_{i, c}$ & $-5\left[^{\circ}\right]$
\end{tabular}

Table 3. Values related to static parameters related to the graphs in Figure 6.

\begin{tabular}{c|c}
\hline$a$ & {$[0.6: 0.9]$} \\
$b$ & {$[0.1: 0.9]$} \\
$\theta$ & {$\left[10^{\circ}: 30^{\circ}\right]$} \\
$T_{i, c}$ & {$\left[-16^{\circ} \mathrm{C}:-2^{\circ} \mathrm{C}\right]$} \\
\hline
\end{tabular}

Table 4. Parameter ranges related to the non-static parameters from Figure 6

\begin{tabular}{|c|c|c|c|c|c|c|c|}
\hline \multirow{9}{*}{ 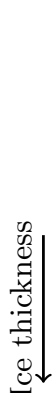 } & & \multicolumn{6}{|c|}{ Air Temperature $\left[{ }^{\circ} \mathrm{C}\right]$} \\
\hline & & \multicolumn{3}{|c|}{$0 \leq T_{a}<5$} & \multicolumn{3}{|c|}{$5 \leq T_{a}<10$} \\
\hline & & \multicolumn{3}{|c|}{ Wind speed $[\mathrm{m} / \mathrm{s}]$} & \multicolumn{3}{|c|}{ Wind speed $[\mathrm{m} / \mathrm{s}]$} \\
\hline & & $0 \leq v<5$ & $5 \leq v<10$ & $v>10$ & $0 \leq v<5$ & $5 \leq v<10$ & $v>10$ \\
\hline & $h=2 \mathrm{~cm}$ & $3-12$ & $2-3$ & $1-2$ & $1-4$ & $1-1$ & $0-1$ \\
\hline & $h=4 \mathrm{~cm}$ & $7-24$ & $4-6$ & $2-4$ & $2-7$ & $1-2$ & $1-1$ \\
\hline & $h=6 \mathrm{~cm}$ & $10-38$ & $6-9$ & $3-5$ & $3-11$ & $2-3$ & $1-2$ \\
\hline & $h=8 \mathrm{~cm}$ & $14-51$ & $8-12$ & $5-7$ & $4-15$ & $2-4$ & $1-2$ \\
\hline & $h=10 \mathrm{~cm}$ & $18-66$ & $10-15$ & $6-10$ & $5-20$ & $3-5$ & $2-3$ \\
\hline
\end{tabular}

Table 5. Estimation of time remaining before ice falls off the cable due to the melting of the ice at its thinnest spot. The values in the table are the estimated number of hours rounded to the nearest hour. 


\begin{tabular}{|c|c|c|c|}
\hline & & \multicolumn{2}{|c|}{ Time of day } \\
\hline & & Morning or afternoon & Midday \\
\hline \multirow{5}{*}{ 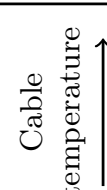 } & $T_{0}=-1^{\circ} \mathrm{C}$ & 3 & 1 \\
\hline & $T_{0}=-4^{\circ} C$ & 11 & 5 \\
\hline & $T_{0}=-7^{\circ} C$ & 18 & 9 \\
\hline & $T_{0}=-10^{\circ} \mathrm{C}$ & 26 & 13 \\
\hline & $T_{0}=-13^{\circ} \mathrm{C}$ & 34 & 17 \\
\hline
\end{tabular}

Table 6. Estimation of time remaining before the ice falls off the cable due to heating of the cable from the sun. The values in the table are the estimated number of hours rounded to the nearest hour.

\section{Conclusion/ Future Work}

This report presented different models for predicting when ice may fall from vertical steel hangers of suspension bridges. We have presented two main categories of models for determining falling times: 1) a model based on heated air, 2) a model based on radiation from the sun. A flow chart was furthermore presented together with tables for determining which model to use and quickly estimating time of failure.

This report has focused on the ice falling problem, however studying the ice formation problem to understand how, when, and what type of ice forms is also an important aspect of the problem that should be considered in future work. Another aspect of the falling problem not considered here is failure due to mechanical stresses due to either wind conditions or traffic. We note however, that the hanger cables are typically heavily damped. The effect of bulk heating of the ice from the sun, as briefly discussed in section 6.4, should also be considered further.

For future work it is expected that the presented models will be verified via physical experiments, conducted either on an actual bridge hanger, or through an experimental setup. In relation to experiments on the bridge we recommend that sensors should be install on the hangers, to collect data for model verification. 
Ice on Suspension Bridge Cables

\begin{tabular}{c|c|c|c|c|c}
\hline Material & $\begin{array}{c}\text { Temp } \\
{[\mathrm{C}]}\end{array}$ & $\begin{array}{c}\text { Density } \\
{\left[\mathrm{kg} / \mathrm{m}^{3}\right]}\end{array}$ & $\begin{array}{c}\text { Thermal Conduct } \\
{[\mathrm{W} / \mathrm{mK}]}\end{array}$ & $\begin{array}{c}\text { Specific Heat } \\
{[\mathrm{kJ} / \mathrm{kgK}]}\end{array}$ & $\begin{array}{c}\text { Linear Expansion } \\
{[\mu \mathrm{m} / \mathrm{m} / \mathrm{K}]}\end{array}$ \\
\hline \multirow{2}{*}{ Ice } & 0 & 916.2 & 2.22 & 2.050 & 50 \\
& -5 & 917.5 & 2.25 & 2.027 & 10 \\
\hline \multirow{2}{*}{ Steel } & 20 & 7900.0 & 40 & 0.470 & 50 \\
\hline
\end{tabular}

Table A 1. Thermal properties of ice and steel used in estimations.

\section{Appendix A Useful data}




\section{References}

[1] Bennani, Lokman, Villedieu, Philippe, \& Salaun, Michel. (2016). A mixed adhesionbrittle fracture model and its application to the numerical study of ice shedding mechanisms. Engineering fracture mechanics, 158, 59-80.

[2] Carslaw., H. S., \& Jaeger, J. C. (1959). Conduction of heat in solids. Oxford University Press.

[3] Crank, J. (1984). Free and moving boundary problems. Oxford University Press.

[4] Druez, Jacques, Louchez, Sylvie, \& McComber, Pierre. (1995). Ice shedding from cables. Cold regions science and technology, 23(4), 377-388.

[5] Fortin, Guy, \& Perron, Jean. (2012). Ice adhesion models to predict shear stress at shedding. Journal of adhesion science and technology, 26(4-5), 523-553.

[6] Gent, R. W., Dart, N. P., \& Cansdale, J. T. (2000). Aircraft icing. Phil. trans. r. soc. lond., a, 2873-2911.

[7] Ghalmi, Z, Menini, R, \& Farzaneh, M. (2009). Theoritical studies and quantification of ice adhesion mechanisms. Pages 8-11 of: Proceedings of the 13th international workshop on atmospheric icing of structures, iwais, andermatt, switzerland.

[8] Guo, Pan, Li, Shengli, \& Wang, Dongwei. (2019). Effects of aerodynamic interference on the iced straddling hangers of suspension bridges by wind tunnel tests. Journal of wind engineering and industrial aerodynamics, 184, 162-173.

[9] Hoffman, Matthew J, Fountain, Andrew G, \& Liston, Glen E. (2014). Near-surface internal melting: a substantial mass loss on antarctic dry valley glaciers. Journal of glaciology, 60(220), 361-374.

[10] Howell, P. Waves and compressible flow, lecture notes, Oxford University, URL: https://courses-archive.maths.ox.ac.uk/node/view_material/48961.

[11] Kietzig, Anne-Marie, Hatzikiriakos, Savvas G, \& Englezos, Peter. (2010). Physics of ice friction. Journal of applied physics, 107(8), 4.

[12] Kleissl, Kenneth, \& Georgakis, CT. (2010). Bridge ice accretion and de-and antiicing systems: A review. Technical university of denmark: Lyngby, denmark.

[13] Kollár, László E, \& Farzaneh, Masoud. (2012). Modeling sudden ice shedding from conductor bundles. Ieee transactions on power delivery, 28(2), 604-611.

[14] Koss, Holger Hundborg, Henningsen, Jesper Frej, \& Olsen, Idar. (2013). Influence of icing on bridge cable aerodynamics. Pages 16-18 of: Proceedings of the 15th international workshop on atmospheric icing of structures (iwais xv), vol. 6.

[15] Makkonen, Lasse. (2012). Ice adhesion-theory, measurements and countermeasures. Journal of adhesion science and technology, 26(4-5), 413-445.

[16] Makkonen, Lasse, \& Tikanmäki, Maria. (2014). Modeling the friction of ice. Cold regions science and technology, 102, 84-93.

[17] Matejicka, Lubomir, Georgakis, Christos Thomas, Schwarz, Andreas, \& Egger, Philipp. (2019). Cable surface for the reduction of risk associated with bridge cable ice accretions. Structural engineering international, 29(3), 425-432.

[18] Matejicka, Lubomir, Georgakis, Christos T, Koss, Holger H, \& Egger, Philipp. (2020). Ice-shedding and aerodynamic investigations of bridge cables with steel wire meshes. Iabse congress - resilient technologies for sustainable infrastructure.

[19] McTavish, Sean, D’Auteuil, Annick, Raeesi, Arash, \& Szilder, Krzysztof. (2021). 
Effect of cable surface geometry and ice accretion shapes on the aerodynamic behaviour of inclined stay cables. Journal of wind engineering and industrial aerodynamics, 216, 104710 .

[20] Myers, Tim G, \& Charpin, Jean PF. (2004). A mathematical model for atmospheric ice accretion and water flow on a cold surface. International journal of heat and mass transfer, 47(25), 5483-5500.

[21] Rønneberg, Sigrid, He, Jianying, \& Zhang, Zhiliang. (2020). The need for standards in low ice adhesion surface research: a critical review. Journal of adhesion science and technology, 34(3), 319-347.

[22] Szilder, Krzysztof, D’Auteuil, Annick, \& McTavish, Sean. (2021). Predicting ice accretion from freezing rain on bridge stay cables. Cold regions science and technology, 187, 103285. 